\title{
Comparing inconsistency of Pairwise Comparison Matrices depending on entries
}

\author{
Bice Cavallo · Alessio Ishizaka · Maria \\ Grazia Olivieri · Massimo Squillante
}

\begin{abstract}
Pairwise comparisons have been a long standing technique for comparing alternatives/criteria and their role has been pivotal in the development of modern decision making methods. Since several types of pairwise comparison matrices (e.g. multiplicative, additive, fuzzy) are proposed in literature, in this paper, we investigate, for which type of matrix, decision makers are more coherent when they express their subjective preferences. By performing an experiment, we found that the additive approach provides the worst level of coherence.
\end{abstract}

Keywords Multi-criteria decision making · Pairwise Comparison Matrix · Consistency evaluation · Abelian linearly ordered group · Behavioral Operations Research

\section{Introduction}

Pairwise comparisons matrices (PCMs) have been long used in psychophysical research to judge and compare sensory intensities (Thurstone, 1927; Gulliksen, 1959). This technique has also gained popularity in decision analysis (Koczkodaj et al (2016) as they are deemed to be more precise than direct judgements (Por and Budescu, 2017, Millet, 1997). In fact, it allows a decision maker to compare two alternatives at a time, thus reducing the complexity of a decisionmaking problem, especially when the set of alternatives is large.

Formally, let $X=\left\{x_{1}, x_{2}, \ldots, x_{n}\right\}$ be a set of decision elements such as criteria or alternatives, the entry $a_{i j}$ of a PCM quantifies the preference intensity of $x_{i}$ over $x_{j}$. In the literature, several types of preference evaluations are considered:

Bice Cavallo

Department of Architecture, University of Naples "Federico II", Italy,

E-mail: bice.cavallo@unina.it

Alessio Ishizaka

Portsmouth Business School, University of Portsmouth, UK

Maria Grazia Olivieri, Massimo Squillante

Department of Law, Economics, Management and Quantitative Methods, University of Sannio, IT 
- if $\left.a_{i j} \in\right] 0,+\infty\left[\right.$ represents a preference ratio, then $A=\left(a_{i j}\right)$ is a multiplicative PCM (Barzilai and Golany, 1990);

- if $\left.a_{i j} \in \mathbb{R}=\right]-\infty,+\infty\left[\right.$ represents a preference difference, then $A=\left(a_{i j}\right)$ is an additive PCM (Barzilai, 1998);

- if $\left.a_{i j} \in\right] 0,1\left[\right.$ reflects a preference degree, then $A=\left(a_{i j}\right)$ is a fuzzy PCM (Tanino, 1984, Chiclana et al, 2009a).

Thus, the entry $a_{i j}$ of a PCM always expresses a relative preference of $x_{i}$ over $x_{j}$; it does not include a measurement unit. As an example, if we have to decide on a car purchase, we express the preference ratio, the preference difference or the preference degree (multiplicative, additive or fuzzy preference, respectively) of one criterion over one other (e.g. price over the fuel consumption) with respect to the selection of a car (we do not compare euro and $k m / l$ but we express subjective preferences).

The foremost used type of evaluation, at least with respect to the number of real-world applications, is the preference ratio, adopted among others by Saaty (1980) in the theory of the Analytic Hierarchy Process (AHP). Several scales have been proposed (Meesariganda and Ishizaka, 2017), but the most popular is the finite scale $\left\{\frac{1}{9}, \frac{1}{8}, \frac{1}{7}, \frac{1}{6}, \frac{1}{5}, \frac{1}{4}, \frac{1}{3}, \frac{1}{2}, 1,2,3,4,5,6,7,8,9\right\}$.

Several techniques have also been proposed to derive the priorities from a PCM (Barzilai, 1997, Cook and Kress, 1988, Jones and Mardle, 2004, Ishizaka and Lusti | 2006: Cavallo and D'Apuzzo, 2012a). However, a priority makes sense only if the decision maker has a minimum level of coherence. For example, if the decision-maker prefers $A$ over $B$ and $B$ over $C$, then, by transitivity, $A$ should be preferred over $C$. If the number of intransitive relations is too high, the calculated priorities are not reliable.

In the literature, it has never been studied for which preference evaluation model (i.e. additive, multiplicative or fuzzy) the decision maker is more (or less) coherent. This paper aims at filling this gap. In particular, we perform an experiment in order to measure the coherence of the participants when they express their subjective preferences by means of additive, multiplicative and fuzzy PCMs.

Experiments have been long used in psychology (Wixted, 2017). Experimental psychology employs human participants to study a variety of topics, including among others sensation and perception, memory, cognition, learning, motivation, emotion. Laboratory experiments have then grown in economics culminating in the Economics Nobel Prize in 2002; it was divided equally between Daniel Kahneman "for having integrated insights from psychological research into economic science, especially concerning human judgement and decisionmaking under uncertainty" and Vernon L. Smith "for having established laboratory experiments as a tool in empirical economic analysis, especially in the study of alternative market mechanisms" (https://www.nobelprize.org/ nobel_prizes/economic-sciences/laureates/2002/). Experiments have also been used in Behavioral Operations Research (White et al, 2017). Keeney et al (1990) asked subjects to provide a direct ranking of alternatives and then to solve the problem with the Multi Attribute Utility (MAU); in a final ranking, 
$80 \%$ changed their initial ranking, mostly in agreement with the MAU ranking. Linares (2009) asked 18 students to rank cars by applying AHP; an automatic algorithm removed the inconsistencies and a new ranking was generated. In a questionnaire, the majority of the students said that when intransitivities were removed, their preferences were not better represented. Huizingh and Vrolijk (1997) asked participants to select a room to rent; they observed that participants were more satisfied with the AHP result than with a random selection. Ishizaka et al (2011) statistically compared the ranking of AHP with three additional rankings given by subject during the experiment: one at the beginning, one after providing the pairwise comparisons and one after learning the ranking provided by AHP. Rankings were found similar and moreover AHP helped the decision-makers to reformulate their rankings by taking into account the suggestions made by AHP. Later, Ishizaka and Siraj (2018) replicate the experiment with another decision problem and the multi-criteria decision methods AHP, MACBETH and SMART. The same observation was found.

In this paper, an experimental approach is also used to compare the three different preference evaluation approaches (i.e. multiplicative, additive and fuzzy) proposed in the literature for dealing with PCMs. Participants have been asked to express subjective preferences about a decision making problem with four criteria and five alternatives; it has been found that the worst level of coherence occurs when participants express preferences differences (e.g. additive approach).

The paper is organized as follows: Section 2 introduces preliminaries useful in the sequel; Section 3 describes the experiment; Section 4 provides results of the experiment and discusses them; Section 5 provides conclusions and future work.

\section{An algebraic approach to Pairwise Comparison Matrices}

Since there is not a unique way for representing the preference intensities under the form of PCM, Cavallo and D'Apuzzo (2009) and Cavallo (2014) propose a unified approach based on Abelian linearly ordered groups (Alo-groups), i.e. commutative groups equipped with an ordering relation. In this context, it is possible to define a consistency condition (i.e. a cardinal transitivity condition of preferences on triplets of decision elements) such that, if it holds, the decision maker is considered fully coherent and his/her judgements are not contradictory (for conditions weaker than consistency see (Cavallo and D'Apuzzo. 2015) and (Cavallo and D'Apuzzo, 2016)). Several authors are following this approach based on Alo-group (e.g. Hou (2016), Xia and Chen (2015), Ramík (2015)). 
2.1 Alo-groups

As the following definition shows, an Alo-group is a commutative group equipped with an ordering relation:

Definition 1 Let $G$ be a non-empty set, $\odot: G \times G \rightarrow G$ a binary operation on $\mathrm{G}, \leq$ a weak order on $G$. Then, $\mathcal{G}=(G, \odot, \leq)$ is an Abelian linearly ordered group (Alo-group for short), if $(G, \odot)$ is an Abelian group and

$$
a \leq b \Rightarrow a \odot c \leq b \odot c .
$$

An isomorphism between two Alo-groups $\mathcal{G}=(G, \odot, \leq)$ and $\mathcal{H}=(H, \circ, \leq)$ is a bijection $\psi: G \rightarrow H$ that is both a lattice isomorphism and a group isomorphism, that is:

$$
a<b \Leftrightarrow \psi(a)<\psi(b) \quad \text { and } \quad \psi(a \odot b)=\psi(a) \circ \psi(b) .
$$

Let us denote with: $e$ the identity element; $a^{(-1)}$ the symmetric of $a$ with respect to $\odot ; \div$ the inverse operation of $\odot$ (i.e. $\left.a \div b=a \odot b^{(-1)}\right)$. By definition, an Alo-group $\mathcal{G}$ is a lattice ordered group (Birkhoff, 1984), that is there exists $\max \{a, b\}$, for each $a, b \in G$; thus, we can consider the following operation, called $\mathcal{G}$-distance, between two elements:

$$
d_{\mathcal{G}}:(a, b) \in G \times G \rightarrow d_{\mathcal{G}}(a, b)=\max \{a \div b, b \div a\} \in G \text {. }
$$

Let $n \in \mathbb{N}_{0}$ and $a \in G$; then, the (n)-natural- power Cavallo and D'Apuzzo, 2009) $a^{(n)}$ of $a \in G$ is:

$$
a^{(n)}=\left\{\begin{array}{lll}
e, & \text { if } & n=0 \\
\bigodot_{i=1}^{n} a, & \text { if } & n \geq 1
\end{array}\right.
$$

\subsubsection{Real continuous Alo-groups}

An Alo-group $\mathcal{G}=(G, \odot, \leq)$ is called continuous if the operation $\odot$ is continuous (Cavallo and D'Apuzzo, 2009), and real if $G$ is a subset of the real line $\mathbb{R}$ and $\leq$ is the weak order on $G$ inherited from the usual order on $\mathbb{R}$. From now on, we will assume that $\mathcal{G}=(G, \odot, \leq)$ is a real continuous Alo-group, with $G$ an open interval. Under these assumptions, the equation $x^{(n)}=a$ has a unique solution (Cavallo and D'Apuzzo, 2009); thus, it is reasonable to consider the following notions of $(n)$-root and $\mathcal{G}$-mean.

Definition 2 (Cavallo and D'Apuzzo, 2009) For each $n \in \mathbb{N}$ and $a \in G$, the $(n)$-root of $a$, denoted by $a^{\left(\frac{1}{n}\right)}$, is the unique solution of the equation $x^{(n)}=a$, that is:

$$
\left(a^{\left(\frac{1}{n}\right)}\right)^{(n)}=a .
$$


Definition 3 Cavallo and D'Apuzzo, 2009) The $\mathcal{G}$-mean $m_{\mathcal{G}}\left(a_{1}, a_{2}, \ldots, a_{n}\right)$ of the elements $a_{1}, a_{2}, \ldots, a_{n}$ of $G$ is

$$
m_{\mathcal{G}}\left(a_{1}, a_{2}, \ldots, a_{n}\right)= \begin{cases}a_{1} & \text { for } \mathrm{n}=1 \\ \left(\bigodot_{i=1}^{n} a_{i}\right)^{(1 / n)} & \text { for } n \geq 2 .\end{cases}
$$

Proposition 1 (Cavallo and D'Apuzzo, 2009) Let $\psi: G \rightarrow H$ be an isomorphism between real continuous Alo-groups $\mathcal{G}=(G, \odot, \leq)$ and $\mathcal{H}=(H, \circ, \leq)$, with $G$ and $H$ open intervals. Then, the following equalities hold:

$$
\begin{aligned}
m_{\mathcal{G}}\left(g_{1}, g_{2}, \ldots, g_{n}\right) & =\psi^{-1}\left(m_{\mathcal{H}}\left(\psi\left(g_{1}\right), \psi\left(g_{2}\right), \ldots, \psi\left(g_{n}\right)\right)\right) ; \\
m_{\mathcal{H}}\left(h_{1}, h_{2}, \ldots, h_{n}\right) & =\psi\left(m_{\mathcal{G}}\left(\psi^{-1}\left(h_{1}\right), \psi^{-1}\left(h_{2}\right), \ldots, \psi^{-1}\left(h_{n}\right)\right)\right) .
\end{aligned}
$$

Examples of real continuous Alo-groups are the following ones:

Multiplicative Alo-group. $\mathcal{R}^{+}=\left(\mathbb{R}^{+}, \cdot, \leq\right)$, where $\cdot$ is the usual multiplication on $\mathbb{R}, e=1$ and $\mathcal{R}^{+}$-mean operator is the geometric mean

$$
m_{\mathcal{R}^{+}}\left(a_{1}, \ldots, a_{n}\right)=\sqrt[n]{\prod_{i=1}^{n} a_{i}}
$$

$\mathcal{R}^{+}$-norm of $a \in \mathbb{R}^{+}$is $\|a\|_{\mathcal{R}^{+}}=\max \left\{a, \frac{1}{a}\right\}, \mathcal{R}^{+}$-distance between $a$ and $b$ is $d_{\mathcal{R}^{+}}(a, b)=\max \left\{\frac{a}{b}, \frac{b}{a}\right\}$.

Additive Alo-group. $\mathcal{R}=(\mathbb{R},+, \leq)$, where + is the usual addition on $\mathbb{R}$, $e=0$ and $\mathcal{R}$-mean operator is the arithmetic mean

$$
m_{\mathcal{R}}\left(a_{1}, \ldots, a_{n}\right)=\frac{\sum_{i=1}^{n} a_{i}}{n}
$$

$\mathcal{R}$-norm of $a \in \mathbb{R}$ is $\|a\|_{\mathcal{R}}=\max \{a,-a\}=|a|$ (i.e. the absolute value of $a), \mathcal{R}$-distance between $a$ and $b$ is $d_{\mathcal{R}}(a, b)=\max \{a-b, b-a\}=|a-b|$.

Fuzzy Alo-group. $\mathcal{I}=(] 0,1[, \otimes, \leq)$, where $\otimes:] 0,1\left[{ }^{2} \rightarrow\right] 0,1[$ is the operation defined by

$$
a \otimes b=\frac{a b}{a b+(1-a)(1-b)},
$$

$e=0.5$ and $\mathcal{I}$-mean operator is given by the following mean:

$$
m_{\mathcal{I}}\left(a_{1}, \ldots, a_{n}\right)=\frac{\sqrt[n]{\prod_{i=1}^{n} a_{i}}}{\sqrt[n]{\prod_{i=1}^{n} a_{i}}+\sqrt[n]{\prod_{i=1}^{n}\left(1-a_{i}\right)}} .
$$

$\mathcal{I}$-norm of $a \in] 0,1\left[\right.$ is $\|a\|_{\mathcal{I}}=\max \{a, 1-a\}, \mathcal{I}$-distance between $a$ and $b$ is the following one:

$$
d_{\mathcal{I}}(a, b)=\max \left\{\frac{a(1-b)}{a(1-b)+(1-a) b}, \frac{b(1-a)}{b(1-a)+(1-b) a}\right\} .
$$


Two real continuous Alo-groups $\mathcal{G}=(G, \odot, \leq)$ and $\mathcal{H}=(H, \circ, \leq)$, with $G$ and $H$ open intervals, are isomorphic (Cavallo and D'Apuzzo, 2009).

Isomorphisms between multiplicative and additive Alo-groups are the following ones:

$$
h: x \in \mathbb{R}^{+} \rightarrow \log x \in \mathbb{R} \quad h^{-1}: y \in \mathbb{R} \rightarrow e^{y} \in \mathbb{R}^{+} .
$$

Isomorphisms between multiplicative and fuzzy Alo-groups are the following ones:

$$
\left.g: t \in \mathbb{R}^{+} \rightarrow \frac{x}{x+1} \in\right] 0,1\left[, \quad g^{-1}: y \in\right] 0,1\left[\rightarrow \frac{y}{1-y} \in \mathbb{R}^{+} .\right.
$$

\subsection{PCMs over real continuous Alo-groups}

Let $\mathcal{G}=(G, \odot, \leq)$ be a real continuous Alo-group, with $G$ an open interval. Let us consider a set $X=\left\{x_{1}, x_{2}, \ldots, x_{n}\right\}$ of decision elements, such as criteria or alternatives, then a PCM over $\mathcal{G}=(G, \odot, \leq)$ is defined as follows:

$$
A=\begin{aligned}
& x_{1} \\
& x_{1} \\
& x_{2} \\
& \vdots \\
& x_{n}
\end{aligned}\left(\begin{array}{cccc}
a_{11} & a_{12} & \ldots & x_{n} \\
a_{21} & a_{22} & \cdots & a_{2 n} \\
\vdots & \vdots & \ddots & \vdots \\
a_{n 1} & a_{n 2} & \cdots & a_{n n}
\end{array}\right),
$$

where $a_{i j}$ quantifies the preference intensity of $x_{i}$ over $x_{j}$. Thus, $a_{i j}=e$ if and only if there is indifference between $x_{i}$ and $x_{j}, a_{i j}>e$ if and only if $x_{i}$ is strictly preferred to $x_{j}$, whereas $a_{i j}<e$ expresses the reverse preference. From now on, we assume that each PCM satisfies $\mathcal{G}$-reciprocity property proposed by Cavallo and D'Apuzzo (2009):

Definition $4 A=\left(a_{i j}\right)$ is a $\mathcal{G}$-reciprocal PCM if verifies the condition:

$$
a_{j i}=a_{i j}^{(-1)} \quad \forall i, j \in\{1, \ldots, n\} .
$$

If the decision maker is fully coherent when he/she expresses his/her preferences by means of a PCM $A=\left(a_{i j}\right)$ defined over $\mathcal{G}$, then $A=\left(a_{i j}\right)$ is $\mathcal{G}$-consistent.

Definition $5 A=\left(a_{i j}\right)$ is a $\mathcal{G}$-consistent PCM, if verifies the following condition:

$$
a_{i k}=a_{i j} \odot a_{j k} \quad \forall i, j, k \in\{1, \ldots, n\} .
$$

$\mathcal{G}$-consistency generalizes several consistency notions proposed in literature, such as multiplicative consistency (Saaty, 1980), additive consistency (Barzilai 1998) and fuzzy multiplicative consistency, called multiplicative transitivity by Tanino (1984, 1988) (see also (Chiclana et al, 2009b; Herrera-Viedma et al 2004)). 
In order to measure how much a PCM is far from $\mathcal{G}$-consistency, Cavallo and D'Apuzzo (2009) propose a consistency index, which is expressed as $\mathcal{G}$ mean of $\mathcal{G}$-distances from $\mathcal{G}$-consistency; i.e. $\mathcal{G}$-mean of $\mathcal{G}$-distances between $a_{i k}$ and $a_{i j} \odot a_{j k}$.

Definition 6 Let $A=\left(a_{i j}\right)$ be a $\mathcal{G}$-reciprocal PCM of order $n \geq 3$. Then, its $\mathcal{G}$-consistency index is:

$$
I_{\mathcal{G}}(A)=\left(\bigodot_{(i, j, k) \in T} d_{\mathcal{G}}\left(a_{i k}, a_{i j} \odot a_{j k}\right)\right)^{\left(\frac{1}{|T|}\right)}
$$

with $T=\{(i, j, k): i<j<k\}$ and $|T|=\frac{n(n-2)(n-1)}{6}$ its cardinality.

Proposition 2 The following statements hold:

1. $I_{\mathcal{G}}(A) \geq e$

2. $I_{\mathcal{G}}(A)=e \Leftrightarrow A$ is $\mathcal{G}$-consistent.

Moreover, $\mathcal{G}$-consistency index satisfies properties proposed by Brunelli (2016) and Brunelli and Fedrizzi (2015) (see Cavallo and D'Apuzzo (2012b)).

Proposition 3 Cavallo and D'Apuzzo, 2009) Let $\mathcal{G}=(G, \odot, \leq)$ and $\mathcal{H}=$ $(H, \circ, \leq)$ be two real continuous Alo-groups, with $G$ and $H$ open intervals, and $\psi: G \rightarrow H$ an isomorphism. Then, for each PCM $A=\left(a_{i j}\right)$ over $\mathcal{G}$, the following equality holds:

$$
\psi\left(I_{\mathcal{G}}(A)\right)=I_{H}(\psi(A))
$$

where $\psi(A)=\left(\psi\left(a_{i j}\right)\right)$.

Remark 1 It is important to note that, Proposition 3 allows us to compare inconsistency of PCMs defined over different Alo-groups (see Example (2) and Example (3)).

\subsubsection{Multiplicative Approach}

Reciprocity is expressed as follows:

$$
a_{j i}=\frac{1}{a_{i j}} \quad \forall i, j \in\{1, \ldots, n\}
$$

Consistency and consistency index in Definition 5 and Definition 6 are expressed as follows:

$$
a_{i k}=a_{i j} \cdot a_{j k} \quad \forall i, j, k \in\{1, \ldots, n\},
$$




$$
\begin{aligned}
I_{\mathcal{R}^{+}}(A) & =\left(\prod_{i=1}^{n-2} \prod_{j=i+1}^{n-1} \prod_{k=j+1}^{n} d_{\mathbb{R}^{+}}\left(a_{i k}, a_{i j} \cdot a_{j k}\right)\right)^{\frac{6}{n(n-2)(n-1)}}= \\
& =\left(\prod_{i=1}^{n-2} \prod_{j=i+1}^{n-1} \prod_{k=j+1}^{n} \max \left\{\frac{a_{i k}}{a_{i j} a_{j k}}, \frac{a_{i j} a_{j k}}{a_{i k}}\right\}\right)^{\frac{6}{n(n-2)(n-1)}} .
\end{aligned}
$$

Example 1 Let us consider the reciprocal multiplicative PCM in (Cavallo and D'Apuzzo, 2009):

$$
A=\left(\begin{array}{llll}
1 & \frac{1}{7} & \frac{1}{7} & \frac{1}{5} \\
7 & 1 & \frac{1}{2} & \frac{1}{3} \\
7 & 2 & 1 & \frac{1}{9} \\
5 & 3 & 9 & 1
\end{array}\right)
$$

thus, its consistency index is the following one:

$$
\begin{aligned}
I_{\mathcal{R}^{+}}(A) & =\sqrt[4]{d_{\mathcal{R}^{+}}\left(a_{13}, a_{12} \cdot a_{23}\right) \cdot d_{\mathcal{R}^{+}}\left(a_{14}, a_{12} \cdot a_{24}\right) \cdot d_{\mathcal{R}^{+}}\left(a_{14}, a_{13} \cdot a_{34}\right) \cdot d_{\mathcal{R}^{+}}\left(a_{24}, a_{23} \cdot a_{34}\right)}= \\
& =\sqrt[4]{2 \cdot 4.2 \cdot 12.6 \cdot 6}=5.02 .
\end{aligned}
$$

\subsubsection{Additive Approach}

Reciprocity is expressed as follows:

$$
a_{j i}=-a_{i j} \quad \forall i, j \in\{1, \ldots, n\} .
$$

Consistency and consistency index in Definition 5 and Definition 6 are expressed as follows:

$$
a_{i k}=a_{i j}+a_{j k} \quad \forall i, j, k \in\{1, \ldots, n\}
$$

$$
\begin{aligned}
I_{\mathcal{R}}(A) & =\frac{6}{n(n-2)(n-1)} \sum_{i=1}^{n-2} \sum_{j=i+1}^{n-1} \sum_{k=j+1}^{n} d_{\mathbb{R}}\left(a_{i k}, a_{i j}+a_{j k}\right)= \\
& =\frac{6}{n(n-2)(n-1)} \sum_{i=1}^{n-2} \sum_{j=i+1}^{n-1} \sum_{k=j+1}^{n}\left(\max \left\{a_{i k}-\left(a_{i j}+a_{j k}\right),\left(a_{i j}+a_{j k}\right)-a_{i k}\right) .\right.
\end{aligned}
$$

Example 2 Let us consider the following reciprocal additive PCM:

$$
B=\left(\begin{array}{cccc}
0 & 2 & 3 & 7 \\
-2 & 0 & 3 & 5 \\
-3 & -3 & 0 & 2 \\
-7 & -5 & -2 & 0
\end{array}\right)
$$


thus, its consistency index is the following one:

$$
\begin{aligned}
I_{\mathcal{R}}(B) & =\frac{d_{\mathcal{R}}\left(a_{13}, a_{12}+a_{23}\right)+d_{\mathcal{R}}\left(a_{14}, a_{12}+a_{24}\right)+d_{\mathcal{R}}\left(a_{14}, a_{13}+a_{34}\right)+d_{\mathcal{R}}\left(a_{24}, a_{23}+a_{34}\right)}{4}= \\
& =\frac{2+0+2+0}{4}=1 .
\end{aligned}
$$

Interestingly, although they are expressed on two different scales, values of inconsistency indices from different representations of preferences are comparable. For instance, by applying the isomorphism in (8), we have that the consistency index of the multiplicative PCM $h^{-1}(B)$ is $I_{R^{+}}\left(h^{-1}(B)\right)=2.72$ that is smaller than $I_{R^{+}}(A)$ in Example 1 thus, $B$ is more consistent than $A$.

\subsubsection{Fuzzy Approach}

Reciprocity is expressed as follows:

$$
a_{j i}=1-a_{i j} \quad \forall i, j \in\{1, \ldots, n\} .
$$

Consistency and consistency index in Definition 5 and Definition 6 are expressed as follows:

$$
\begin{gathered}
a_{i k}=\frac{a_{i j} \cdot a_{j k}}{a_{i j} \cdot a_{j k}+\left(1-a_{i j}\right) \cdot\left(1-a_{j k}\right)} \quad \forall i, j, k \in\{1, \ldots, n\}, \\
I_{\mathcal{I}}(A)=\frac{a}{a+b}
\end{gathered}
$$

with

$$
\begin{aligned}
a & =\left(\prod_{i=1}^{n-2} \prod_{j=i+1}^{n-1} \prod_{k=j+1}^{n} d_{\mathcal{I}}\left(a_{i k}, a_{i j} \otimes a_{j k}\right)\right)^{\frac{6}{n(n-2)(n-1)}}= \\
& =\left(\prod_{i=1}^{n-2} \prod_{j=i+1}^{n-1} \prod_{k=j+1}^{n} \max \left\{\frac{a_{i k}\left(1-\left(a_{i j} \otimes a_{j k}\right)\right)}{a_{i k}\left(1-\left(a_{i j} \otimes a_{j k}\right)\right)+\left(1-a_{i k}\right)\left(a_{i j} \otimes a_{j k}\right)}, \frac{\left(a_{i j} \otimes a_{j k}\right)\left(1-a_{i k}\right)}{\left(a_{i j} \otimes a_{j k}\right)\left(1-a_{i k}\right)+\left(1-\left(a_{i j} \otimes a_{j k}\right)\right) a_{i k}}\right\}\right)^{\frac{6}{n(n-2)(n-1)}}
\end{aligned}
$$

and

$$
\begin{aligned}
b & =\left(\prod_{i=1}^{n-2} \prod_{j=i+1}^{n-1} \prod_{k=j+1}^{n}\left(1-d_{\mathcal{I}}\left(a_{i k}, a_{i j} \otimes a_{j k}\right)\right)\right)^{\frac{6}{n(n-2)(n-1)}}= \\
& =\left(\prod_{i=1}^{n-2} \prod_{j=i+1}^{n-1} \prod_{k=j+1}^{n} 1-\max \left\{\frac{a_{i k}\left(1-\left(a_{i j} \otimes a_{j k}\right)\right)}{a_{i k}\left(1-\left(a_{i j} \otimes a_{j k}\right)\right)+\left(1-a_{i k}\right)\left(a_{i j} \otimes a_{j k}\right)}, \frac{\left(a_{i j} \otimes a_{j k}\right)\left(1-a_{i k}\right)}{\left(a_{i j} \otimes a_{j k}\right)\left(1-a_{i k}\right)+\left(1-\left(a_{i j} \otimes a_{j k}\right)\right) a_{i k}}\right\}\right)^{\frac{6}{n(n-2)(n-1)}}
\end{aligned}
$$

Example 3 Let us consider the following reciprocal fuzzy PCM:

$$
C=\left(\begin{array}{cccc}
0.5 & 0.67 & 0.86 & 0.67 \\
0.33 & 0.5 & 0.75 & 0.33 \\
0.14 & 0.25 & 0.5 & 0.14 \\
0.33 & 0.67 & 0.86 & 0.5
\end{array}\right)
$$

Its consistency index can be computed by applyng isomorphisms in (9); thus, we have:

$$
I_{\mathcal{I}}(C)=g\left(I_{\mathcal{R}^{+}}\left(g^{-1}(C)\right)\right)=0.59 .
$$

We stress that $I_{\mathcal{R}^{+}}\left(g^{-1}(C)\right)=1.41$ is smaller than both $I_{\mathcal{R}^{+}}(A)$ in Example 1 and $I_{R^{+}}\left(h^{-1}(B)\right)$ in Example 2 thus $C$ is more consistent than both $A$ and $B$. 


\section{The experiment}

In order to measure the inconsistency of different types of PCMs, we perform an experiment; we use an opinion survey with a sample of 120 students of Department of Architecture of University of Naples "Federico II ". The students completed the survey during a class period and received instructions to fill out it. On average, the experiment lasted one hour.

In a problem inspired by an example provided by Saaty (1987), participants were asked to choose their preferred university based on the following four criteria:

$C_{1}$. Distance from Napoli. As far as distance is concerned, the farther away from Napoli an university is perceived to be better (but not necessarily linearly);

$C_{2}$. Reputation of the University. It concerns how the university is rated;

$C_{3}$. Number of students in a class. It is assumed that small classes are preferred to large ones because more attention is ensured to the students;

$C_{4}$. Attractiveness of the city. It concerns how happy the participant felt at the city.

Thus, participants were asked to compare pairwise the criteria with respect to the selection of a University and to compare pairwise five universities on the set $X=\left\{x_{1}=\right.$ University of Sannio, $x_{2}=$ University "La Sapienza", $x_{3}=$ University of Bologna, $x_{4}=$ Politecnico Milano, $x_{5}=$ University of Barcelona $\}$ with respect to each criterion. Hierarchy of the problem is shown in Figure 1.

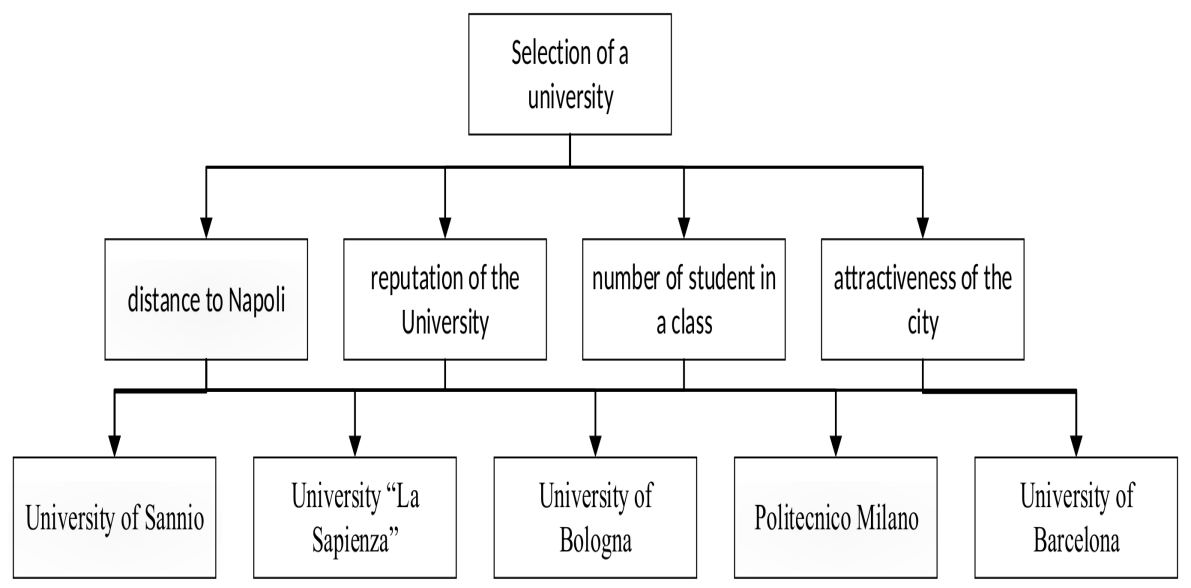

Fig. 1 Hierarchy of the problem

The goal of this experimentation is to evaluate the coherence of the participants by means of the following approaches: 
M. The pairwise comparisons are expressed as preference ratio on the interval ] $0,+\infty[$ (i.e. multiplicative approach on $] 0,+\infty[$ );

S. The pairwise comparisons are expressed as preference ratio on Saaty scale $\left\{\frac{1}{9}, \frac{1}{8}, \frac{1}{7}, \frac{1}{6}, \frac{1}{5}, \frac{1}{4}, \frac{1}{3}, \frac{1}{2}, 1,2,3,4,5,6,7,8,9\right\}$ (i.e. multiplicative approach on Saaty scale; we stress that, although there is not an Alo-group defined on Saaty scale, since multiplicative PCMs over Saaty scale are used in several real world applications, they are also considered in our experiment);

A. The pairwise comparisons are expressed as preference difference on the interval ] $-\infty,+\infty[$ (i.e. additve approach);

F. The pairwise comparisons are expressed as a preference degree on the interval ]0,1[ (i.e. fuzzy approach).

Thus, we built four opinion surveys $Q^{M}, Q^{S}, Q^{A}$ and $Q^{F}$; each $Q^{j}$, with $j \in\{M, S, A, F\}$, includes five questionnaires:

- $Q_{0}^{j}$ for pairwise comparing criteria $C_{1}, C_{2}, C_{3}$, and $C_{4}$ with respect to the goal (i.e. the selection of a university);

- $Q_{1}^{j}$ for pairwise comparing universities in $X$ with respect to $C_{1}$;

- $Q_{2}^{j}$ for pairwise comparing universities in $X$ with respect to $C_{2}$;

- $Q_{3}^{j}$ for pairwise comparing universities in $X$ with respect to $C_{3}$;

- $Q_{4}^{j}$ for pairwise comparing universities in $X$ with respect to $C_{4}$.

In the design of the experiment, we decided that each participant is allowed to complete only one survey. This "between-subject" designed experiment is more appropriate for our purpose than a "within-subject" designed experiment, where participants are exposed to more than one survey. It has been long observed that within-subject cannot be used when independence of multiple exposure is not warranty. Poulton (1973) found that within-subject experiments have a range effect. In fact, the respondents have a reference point when they are responding the second survey. Greenwald (1976) criticizes within-subject designs based on the effects of practice, sensitization and carryover that confound causality. The reader can refer to Charness et al (2012) for further details about the two experimental methods, and to Ishizaka and Siraj (2018) for a recent application of the between-subject method. In particular, Ishizaka and Siraj (2018) evaluated three multi-criteria decision-making tools where each participant used only one tool. In addition, the statistical units included in the sample come from the same population (homogeneous for age and level degree); thus, a low within group variability is expected. Thus, we have that:

- survey $Q^{M}$, including questionnaires $Q_{0}^{M}, Q_{1}^{M}, Q_{2}^{M}, Q_{3}^{M}$ and $Q_{4}^{M}$, is given to 30 participants $m_{1}, \ldots m_{30}$ and we asked them to express their preference ratios on $] 0,+\infty\left[\right.$. In this way, for each participant $m_{k}$, with $k \in\{1, \ldots 30\}$, we obtained 5 multiplicative PCMs $M_{0}^{k}, M_{1}^{k}, M_{2}^{k}, M_{3}^{k}$ and $M_{4}^{k}$ on $] 0,+\infty[$; - survey $Q^{S}$, including questionnaires $Q_{0}^{S}, Q_{1}^{S}, Q_{2}^{S}, Q_{3}^{S}$ and $Q_{4}^{S}$, is given to 30 participants $s_{1}, \ldots s_{30}$ and we asked them to express their preference ratios on Saaty scale. In this way, for each participant $s_{k}$, with $k \in\{1, \ldots 30\}$, we obtained 5 multiplicative PCMs $S_{0}^{k}, S_{1}^{k}, S_{2}^{k}, S_{3}^{k}$ and $S_{4}^{k}$ on Saaty scale; 
- survey $Q^{A}$, including questionnaires $Q_{0}^{A}, Q_{1}^{A}, Q_{2}^{A}, Q_{3}^{A}$ and $Q_{4}^{A}$, is given to 30 participants $a_{1}, \ldots a_{30}$ and we asked them to express their preference differences on $]-\infty,+\infty$ [. In this way, for each participant $a_{k}$, with $k \in$ $\{1, \ldots 30\}$, we obtained 5 additive PCMs $A_{0}^{k}, A_{1}^{k}, A_{2}^{k}, A_{3}^{k}$ and $A_{4}^{k}$ on ]$\infty,+\infty[$

- survey $Q^{F}$, including questionnaires $Q_{0}^{F}, Q_{1}^{F}, Q_{2}^{F}, Q_{3}^{F}$ and $Q_{4}^{F}$, is given to 30 participants $f_{1}, \ldots f_{30}$ and we asked them to express their preference degrees on $] 0,1\left[\right.$. In this way, for each participant $f_{k}$, with $k \in\{1, \ldots 30\}$, we obtained 5 fuzzy PCMs $F_{0}^{k}, F_{1}^{k}, F_{2}^{k}, F_{3}^{k}$ and $F_{4}^{k}$ on $] 0,1[$.

As an example, Figure 2 shows questionnaire $Q_{2}^{M}$; we stress that the preference ratios are chosen in $[1,+\infty[$ and not in $] 0,+\infty[$, because, for each pair of universities, we ask to choose the preferred one; the remaining entries of the related PCMs are computed by means of reciprocity.

We stress that each pairwise comparison expresses a subjective relative preference of a decision element over one other and it does not include a measurement unit; it is a preference ratio, a preference difference or a preference degree (e.g. preference of $C_{1}$ over $C_{2}$ with respect to selection of a university, or the preference of $x_{1}$ over $x_{2}$ with respect to the reputation of the University).

Please, for each pair of universities, select the preferred university with respect to its reputation and write your preference ratio by choosing a number in $[1,+\infty[$. If you are indifferent between two universities, you have to write a preference ratio equal to 1 and to select both the universities.

E.g. a preference ratio equal to 3.5 means that the selected university is preferred three times and half with respect to the other one.

$\begin{array}{lll}\text { University of Sannio } & \text { "Sapienza" of Roma } & \text { Preference ratio }= \\ \text { University of Sannio } & \text { University of Bologna } & \text { Preference ratio }= \\ \text { University of Sannio } & \text { Politecnico of Milano } & \text { Preference ratio }= \\ \text { University of Sannio } & \text { University of Barcellona } & \text { Preference ratio }= \\ \text { "Sapienza" of Roma } & \text { University of Bologna } & \text { Preference ratio }= \\ \text { "Sapienza" of Roma } & \text { Politecnico of Milano } & \text { Preference ratio }= \\ \text { "Sapienza" of Roma } & \text { University of Barcellona } & \text { Preference ratio }= \\ \text { University of Bologna } & \text { Politecnico of Milano } & \text { Preference ratio }= \\ \text { University of Bologna } & \text { University of Barcellona } & \text { Preference ratio }= \\ \text { Politecnico of Milano } & \text { University of Barcellona } & \text { Preference ratio }=\end{array}$

Fig. 2 Questionnaire $Q_{2}^{M}$ for comparing the five universities with respect to criterion $C_{2}$ by means of the multiplicative approach.

In order to establish for which type of PCM the participants are more coherent when they express subjective preferences, firstly, for each $i \in\{0,1,2,3,4\}$, we computed the following means of consistency indices:

$$
\begin{gathered}
m_{\mathcal{R}^{+}}\left(I_{\mathcal{R}^{+}}\left(M_{i}^{1}\right) \ldots, I_{\mathcal{R}^{+}}\left(M_{i}^{k}\right) \ldots, I_{\mathcal{R}^{+}}\left(M_{i}^{30}\right)\right) \\
m_{\mathcal{R}^{+}}\left(I_{\mathcal{R}^{+}}\left(S_{i}^{1}\right) \ldots, I_{\mathcal{R}^{+}}\left(S_{i}^{k}\right) \ldots, I_{\mathcal{R}^{+}}\left(S_{i}^{30}\right)\right)
\end{gathered}
$$




$$
\begin{gathered}
m_{\mathcal{R}}\left(I_{\mathcal{R}}\left(A_{i}^{1}\right) \ldots, I_{\mathcal{R}}\left(A_{i}^{k}\right) \ldots, I_{\mathcal{R}}\left(A_{i}^{30}\right)\right) \\
I_{i}^{F}=m_{\mathcal{I}}\left(I_{\mathcal{I}}\left(F_{i}^{1}\right) \ldots, I_{\mathcal{I}}\left(F_{i}^{k}\right) \ldots, I_{\mathcal{I}}\left(F_{i}^{30}\right)\right) .
\end{gathered}
$$

By item 1. in Proposition 2, we have that $(18)$ and $(19)$ are geometric means (5) in $[1,+\infty[, 220)$ is an arithmetic mean (6) in $[0,+\infty$ [ and (21) is a fuzzy $\mathcal{I}$-mean 77$]$ in $[0.5,1[$. Thus, in order to compare the inconsistencies of PCMs defined over different Alo-groups, by Proposition 1 and isomorphisms in (8) and (9), we obtain the following fuzzy $\mathcal{I}$-means on the same interval [0.5, 1:

$$
\begin{gathered}
I_{i}^{M}=g\left(m_{\mathcal{R}^{+}}\left(I_{\mathcal{R}^{+}}\left(M_{i}^{1}\right) \ldots, I_{\mathcal{R}^{+}}\left(M_{i}^{k}\right) \ldots, I_{\mathcal{R}^{+}}\left(M_{i}^{30}\right)\right)\right) \\
I_{i}^{S}=g\left(m_{\mathcal{R}^{+}}\left(I_{\mathcal{R}^{+}}\left(S_{i}^{1}\right) \ldots, I_{\mathcal{R}^{+}}\left(S_{i}^{k}\right) \ldots, I_{\mathcal{R}^{+}}\left(S_{i}^{30}\right)\right)\right) \\
I_{i}^{A}=g\left(h^{-1}\left(m_{\mathcal{R}}\left(I_{\mathcal{R}}\left(A_{i}^{1}\right) \ldots, I_{\mathcal{R}}\left(A_{i}^{k}\right) \ldots, I_{\mathcal{R}}\left(A_{i}^{30}\right)\right)\right)\right)
\end{gathered}
$$

\section{Results and discussion}

The results of the experiment described in the previous section are shown in Table 1] where:

- column $I^{M}$ contains $I_{0}^{M}, I_{1}^{M}, I_{2}^{M}, I_{3}^{M}$ and $I_{4}^{M}$ in (22);

- column $I^{S}$ contains $I_{0}^{S}, I_{1}^{S}, I_{2}^{S}, I_{3}^{S}$ and $I_{4}^{S}$ in (23);

- column $I_{i}^{A}$ contains $I_{0}^{A}, I_{1}^{A}, I_{2}^{A}, I_{3}^{A}$ and $I_{4}^{A}$ in (24);

- column $I^{F}$ contains $I_{0}^{F}, I_{1}^{F}, I_{2}^{F}, I_{3}^{F}$ and $I_{4}^{F}$ in (21);

- column $p$ contains $p$ value of ANOVA (ANalysis Of VAriance) test;

- column $F$ contains $F$ value of ANOVA test.

\begin{tabular}{|c|c|c|c|c|c|c|}
\hline & $I^{M}$ & $I^{S}$ & $I^{A}$ & $I^{F}$ & $p$ & $F$ \\
\hline$i=0$ & 0.69 & 0.76 & 0.96 & 0.72 & $2.48 \mathrm{E}-8$ & 15.15 \\
\hline$i=1$ & 0.72 & 0.76 & 0.99 & 0.73 & $3.28 \mathrm{E}-10$ & 19.43 \\
\hline$i=2$ & 0.73 & 0.77 & 0.98 & 0.73 & 0.00143 & 5.52 \\
\hline$i=3$ & 0.7 & 0.73 & 0.98 & 0.71 & 0.00083 & 5.96 \\
\hline$i=4$ & 0.78 & 0.76 & 0.99 & 0.73 & 0.00004 & 8.59 \\
\hline
\end{tabular}

Table 1 Means of consistency indices of the PCMs built by surveys $Q^{M}, Q^{S}, Q^{A}, Q^{F}$ and ANOVA results.

It can be seen from Table 1 that the multiplicative case $\left(I^{M}\right)$ is the least inconsistent in four cases $(i=0,1,2,3)$ out of five; we recall that $I^{M}, I^{S}, I^{A}$ and $I^{F}$ assume value in $[0.5,1[$ and the greater their value the more inconsistency is. The most inconsistent is always the additive case $\left(I^{A}\right)$. These differences are significant with an ANOVA test at a confidence threshold $p=0.05$; indeed, $p$ value is always smaller than the confidence threshold and $F$ value is always higher than critical value $F_{\text {crit }}=2.69$. However, if we remove the additive case (Table 2), then the differences are not anymore significant at $\mathrm{p}=0.05$; indeed, all $p$ values are higher than the confidence threshold and the $F$ values are 


\begin{tabular}{|c|c|c|c|c|c|}
\hline & $I^{M}$ & $I^{S}$ & $I^{F}$ & $p$ & $F$ \\
\hline$i=0$ & 0.69 & 0.76 & 0.72 & 0.067 & 2.79 \\
\hline$i=1$ & 0.72 & 0.76 & 0.73 & 0.132 & 2.07 \\
\hline$i=2$ & 0.73 & 0.77 & 0.73 & 0.058 & 2.94 \\
\hline$i=3$ & 0.7 & 0.73 & 0.71 & 0.287 & 1.26 \\
\hline$i=4$ & 0.78 & 0.76 & 0.73 & 0.496 & 0.70 \\
\hline
\end{tabular}

Table 2 Means of consistency indices of the PCMs built by surveys $Q^{M}, Q^{S}, Q^{F}$ and ANOVA results.

smaller than the critical value $F_{\text {crit }}=3.10$.

The additive method is therefore to be avoided for a better consistency.

\section{Conclusions and future work}

In this paper, an experiment is performed in order to compare, by a behavioral point of view, three different preference evaluation approaches proposed in literature for dealing with PCMs; i.e. multiplicative, additive and fuzzy approach.

Multiplicative, additive and fuzzy preferences share the same algebraic structure (Cavallo and D'Apuzzo, 2009), i.e. Alo-group. Alo-groups and related isomorphisms are necessary to show the formal equivalence between different representations and to naturally extend concepts and properties from one representation to another one. By quoting Fraleigh (2002), an isomorphism is defined as "the concept of two systems being structurally identical". In this paper, we use properties of Alo-groups and related isomorphisms for comparing inconsistency of multiplicative, additive and fuzzy PCMs, in a real decision making problem.

By means of opinion surveys, participants have been asked to express subjective preferences about a decision making problem with four criteria and five alternatives; the experiment shows that by expressing "preference ratios" (i.e. multiplicative preferences) or "preference degrees" (i.e. fuzzy preferences), the participants are more coherent than when they express "preference differences" (i.e. additive preferences).

Of course, participants when they express their preferences have not in mind the isomorphisms and, moreover, it could be that the notion of "preference difference" has a less understandable meaning with respect to "preference ratio" or "preference degree". Thus, Behavioral Operations Research is an interesting approach when human behavior needs to be examined and taken into consideration.

Our future work will be directed to apply our experimental approach to other decision problems. Moreover, we plan to carry out similar experiments in order to analyze conditions weaker than consistency, i.e. a cardinal transitivity, in both individual and collective decision procedures, such as ordinal transitivity (Cavallo and D'Apuzzo, 2015), that is the minimal logical requirement 
that decision maker's preferences should satisfy, and weak consistency (Cavallo and D'Apuzzo, 2016) that ensures reliability to a priority vector proposed by Cavallo and D'Apuzzo (2012a).

\section{References}

Barzilai J (1997) Deriving weights from pairwise comparison matrices. The Journal of the Operational Research Society 48(12):1226-1232

Barzilai J (1998) Consistency measures for pairwise comparison matrices. Journal of Multi-Criteria Decision Analysis 7(3):123-132

Barzilai J, Golany B (1990) Deriving weights from pairwise comparison matrices: The additive case. Operations Research Letters 9(6):407 - 410

Birkhoff G (1984) Lattice theory, vol 25. American Mathematical Soc.

Brunelli M (2016) Studying a set of properties of inconsistency indices for pairwise comparisons. Annals of Operations Research

Brunelli M, Fedrizzi M (2015) Axiomatic properties of inconsistency indices for pairwise comparisons. Journal of the Operational Research Society 66(1):115

Cavallo B (2014) A further discussion of a semiring-based study of judgment matrices: properties and models? [information sciences 181 (2011) 21662176]. Information Sciences 287:61 - 67

Cavallo B, D'Apuzzo L (2009) A general unified framework for pairwise comparison matrices in multicriterial methods. International Journal of Intelligent Systems 24(4):377-398

Cavallo B, D'Apuzzo L (2012a) Deriving weights from a pairwise comparison matrix over an alo-group. Soft Computing 16(2):353-366

Cavallo B, D'Apuzzo L (2012b) Investigating properties of the $\odot$-consistency index. In: Advances in Computational Intelligence, Springer, pp 315-327

Cavallo B, D'Apuzzo L (2015) Reciprocal transitive matrices over abelian linearly ordered groups: Characterizations and application to multi-criteria decision problems. Fuzzy Sets and Systems 266:33-46

Cavallo B, D'Apuzzo L (2016) Ensuring reliability of the weighting vector: Weak consistent pairwise comparison matrices. Fuzzy Sets and Systems 296:21-34

Charness G, Gneezy U, Kuhn MA (2012) Experimental methods: Betweensubject and within-subject design. Journal of Economic Behavior \& Organization $81(1): 1-8$

Chiclana F, Herera-Viedma E, Alonso S, Herera F (2009a) Cardinal consistency of reciprocal preference relations: A characterization of multiplicative transitivity. IEEE Transaction on fuzzy sistems 17(1):14-23

Chiclana F, Herrera-Viedma E, Alonso S, Herrera F (2009b) Cardinal consistency of reciprocal preference relations: A characterization of multiplicative transitivity. Fuzzy Systems, IEEE Transactions on 17(1):14-23 
Cook WD, Kress M (1988) Deriving weights from pairwise comparison ratio matrices: An axiomatic approach. European Journal of Operational Research $37(3): 355-362$

Fraleigh JB (2002) A First Course in Abstract Algebra, 7th edn. Pearson

Greenwald A (1976) Within-subjects designs: to use or not to use. Psychological Bulletin 83(2):314-320

Gulliksen H (1959) Mathematical solutions for psychological problems. American Scientist 47:178-201

Herrera-Viedma E, Herrera F, Chiclana F, Luque M (2004) Some issues on consistency of fuzzy preference relations. European Journal of Operational Research 154(1):98 - 109

Hou F (2016) A multiplicative alo-group based hierarchical decision model and application. Communications in Statistics - Simulation and Computation $45(8)$

Huizingh EK, Vrolijk HC (1997) A comparison of verbal and numerical judgments in the analytic hierarchy process. Organizational Behavior and $\mathrm{Hu}-$ man Decision Processes 70(3):237 - 247

Ishizaka A, Lusti M (2006) How to derive priorities in ahp: a comparative study. Central European Journal of Operations Research 14(4):387-400

Ishizaka A, Siraj S (2018) Are multi-criteria decision-making tools useful? an experimental comparative study of three methods. European Journal of Operational Research 264(2):462 - 471

Ishizaka A, Balkenborg D, Kaplan T (2011) Does ahp help us make a choice? an experimental evaluation. Journal of the Operational Research Society 62(10):1801-1812

Jones DF, Mardle SJ (2004) A distance-metric methodology for the derivation of weights from a pairwise comparison matrix. Journal of the Operational Research Society 55(8):869-875

Keeney RL, von Winterfeldt D, Eppel T (1990) Eliciting public values for complex policy decisions. Management Science 36(9):1011-1030

Koczkodaj W, Mikhailov L, Redlarski G, Soltys M, Szybowski J, Tamazian G, Wajch E, Yuen KKF (2016) Important facts and observations about pairwise comparisons (the special issue edition). Fundamenta Informaticae 144(3-4):291-307

Linares P (2009) Are inconsistent decisions better? an experiment with pairwise comparisons. European Journal of Operational Research 193(2):492 498

Meesariganda BR, Ishizaka A (2017) Mapping verbal ahp scale to numerical scale for cloud computing strategy selection. Applied Soft Computing 53:111 $-118$

Millet I (1997) The effectiveness of alternative preference elicitation methods in the analytic hierarchy process. Journal of Multi-Criteria Decision Analysis 6(1):41-51

Por HH, Budescu DV (2017) Eliciting subjective probabilities through pairwise comparisons. Journal of Behavioral Decision Making 30(2):181-196 
Poulton EC (1973) Unwanted range effects from using within-subject experimental designs. Psychological Bulletin 80(2):113-121

Ramík J (2015) Pairwise comparison matrix with fuzzy elements on alo-group. Information Sciences 297:236-253

Saaty R (1987) The analytic hierarchy process-what it is and how it is used. Mathl Modelling 9(3-5):161-176

Saaty TL (1980) The Analytic Hierarchy Process. McGraw-Hill, New York

Tanino T (1984) Fuzzy preference orderings in group decision making. Fuzzy Sets and Systems 12(2):117 - 131

Tanino T (1988) Fuzzy preference relations in group decision making, Springer, Heidelberg, pp 54-71. Non-Conventional Preference Relations in DecisionMaking

Thurstone L (1927) A law of comparative judgments. Psychological Review $34(4): 273-286$

White L, Kunc M, White L, Malpass J, Kunc M (eds) (2017) Behavioral Operational Research - Theory, Methodology and Practice. Springer

Wixted J (2017) Stevens' Handbook of Experimental Psychology and Cognitive Neuroscience. Wiley

Xia M, Chen J (2015) Consistency and consensus improving methods for pairwise comparison matrices based on abelian linearly ordered group. Fuzzy Sets and Systems 266(0):1 - 32 